\title{
De quoi les Conti sont-ils le nom ?
}

Radicalisation des luttes sociales et restructurations financières

What are the Conti the name of? Radicalisation of social struggles and financial restructurings

\section{Pascal Depoorter et Nathalie Frigul}

\section{(2) OpenEdition}

Journals

Édition électronique

URL : http://journals.openedition.org/travailemploi/6234

DOI : 10.4000/travailemploi.6234

ISSN : 1775-416X

Éditeur

DARES - Ministère du Travail

\section{Édition imprimée}

Date de publication : 15 mars 2014

Pagination : $51-68$

ISSN : 0224-4365

\section{Référence électronique}

Pascal Depoorter et Nathalie Frigul, « De quoi les Conti sont-ils le nom ? », Travail et Emploi [En ligne],

137 | janvier-mars 2014, mis en ligne le 01 janvier 2016, consulté le 30 avril 2019. URL : http:// journals.openedition.org/travailemploi/6234; DOI : 10.4000/travailemploi.6234 


\title{
De quoi les Conti sont-ils le nom ? Radicalisation des luttes sociales et restructurations financières
}

\author{
Pascal Depoorter ${ }^{(*)}$, Nathalie Frigul(**)
}

Cet article étudie l'un des mouvements sociaux les plus emblématiques de l'année 2009, qui s'est déroulé dans l'Oise à l'usine Continental. À partir d'une recherche encore en cours, les auteurs essayent d'appréhender les façons dont cette lutte sociale s'est construite et s'est radicalisée. Dans un premier temps, ils la resituent dans l'histoire de l'usine, des conflits antérieurs, et examinent les conditions de l'annonce de la fermeture. Ensuite, les auteurs s'intéressent aux modalités et aux moyens donnés à la lutte en se centrant sur les acteurs du mouvement. Ils analysent ainsi les éléments les plus marquants d'une critique sociale et des formes de politisation qu'elle engage. Enfin, ils tentent de saisir les ressorts de la mobilisation collective qui reposent sur un double registre, matériel et symbolique : indemnisation financière de la perte d'emploi et de salaire et réparation de la violence sociale subie à la suite d'une fermeture qui a été vécue sur le mode de l'injustice.

Depuis les manifestations musclées des sidérurgistes lorrains du début des années 1980 (Montlibert, 1989), le temps n'était plus, semblet-il, aux luttes mais à l'adaptation de l'appareil de production à la mondialisation (ASKENAZY, 2011). En dépit de quelques mouvements épars (BÉROUD, Mouriaux, 2001), les décennies 1980 et 1990 marquent un recul assez net des grèves ouvrières. Néanmoins, de nouvelles formes d'action collective apparaissent, se caractérisant davantage par des pétitions, des débrayages et des arrêts de travail plus courts que par le passé (Béroud et al., 2008).

$\mathrm{Au}$ tournant des années 2000, quelques mouvements sociaux qui attirent l'attention des journalistes sont perçus comme des actes désespérés pour le maintien de l'emploi. L'entreprise Cellatex dans les Ardennes a ainsi occupé la scène médiatique pendant plusieurs semaines en juillet 2000 (Larose et al., 2001). Pour se faire entendre, les ouvriers de cette petite usine avaient menacé de déverser de l'acide dans la Meuse. L'action militante se situait dans la continuité des modes virils des mobilisations populaires et prenait en même temps une forme réactualisée. Ces conflits sont mis en scène et jouent sur les symboles, inspirés sans doute par les «nouveaux mouvements sociaux»

(*) CURAPP-ESS UMR 7319 (Centre universitaire de recherches sur l'action publique et le politique, épistémologie et sciences sociales), université de Picardie Jules-Verne; pascal.depoorter@u-picardie.fr

(**) CURAPP-ESS UMR 7319 (Centre universitaire de recherches sur l'action publique et le politique, épistémologie et sciences sociales), université de Picardie Jules-Verne; nathalie.frigul@u-picardie.fr dont la particularité est d'être portés par des militants très au fait des modes d'accès au traitement médiatique (MATHIEU, 2011). Présentés auparavant dans la rubrique des faits divers, les fermetures d'entreprises et plans sociaux alimentent dès lors les pages politiques et sociales de la presse locale et aussi nationale(1). Ce renouveau des mouvements sociaux semble, à bien des égards, annoncer le retour au premier plan de la question ouvrière. Temps fort de la protestation sociale, le conflit qui se développe à Continental Clairoix au printemps 2009 constitue un épisode récent de telles luttes. À la fois protestataire et porteur d'une critique sociale, il se traduit par des actions «coup-depoing», dont la destruction des éléments de mobilier à la sous-préfecture de Compiègne a été le point d'orgue. Comment expliquer le caractère violent et «radical» de la lutte des Conti ?

Cette lutte s'inscrit tout d'abord dans les premières répercussions de la crise financière et

(1) La liquidation des sites industriels, sans qu'il y ait pour autant dépôt de bilan, fait ainsi une brève apparition au début de la campagne présidentielle de 2002 quand le journal de vingt heures de TF1 montre les images de Lionel Jospin aux prises avec un syndicaliste de Lu. La défaite électorale du candidat socialiste est imputée à la défection du vote des classes populaires dont Le Monde tente de saisir les contours quelques mois plus tard dans un dossier documenté, «La France des oubliés», autrement dit la France des classes populaires qui subissent le plus durement les effets des mutations économiques. Depuis, elles constituent un enjeu de communication politique qui met la question du travail au cœur de la campagne présidentielle de 2007 et place le déclin industriel au centre de celle de 2012. 
bancaire qui a éclaté en septembre $2008^{(2)}$. L'année 2009 est marquée par une augmentation d'une ampleur sans précédent du nombre de fermetures d'entreprises, phénomène qui se traduit par un recul historique de l'emploi en France(3). Au moment où se développe le conflit social à l'usine Continental, début mars, des milliers de personnes sont en train de perdre leur travail. Les intérimaires et les salariés en situation précaire qui constituent traditionnellement «la variable d'ajustement» du marché de l'emploi sont touchés les premiers. Mais l'annonce de la fermeture de l'usine Continental se distingue des cas majoritaires de faillites, car cette unité de production moderne affiche des taux annuels de productivité honorables et dégage un résultat bénéficiaire en 2008. Par la condamnation d'un de ses sites français, le groupe allemand entend, se justifie-t-il, anticiper les effets de la «crise» sur le marché du pneumatique en raison notamment du ralentissement de la production automobile. Si cette décision est incompréhensible pour les salariés locaux, elle ne permet pourtant pas à elle seule d'expliquer l'ampleur et la forme de ce conflit social très médiatisé.

Le contexte politique national du début de l'année 2009 entre aussi en ligne de compte. Il est caractérisé par de fortes mobilisations pour la défense du pouvoir d'achat et de l'emploi. En janvier et en mars, contestant la politique gouvernementale, des centaines de milliers de manifestants battent le pavé à l'appel de l'ensemble des confédérations syndicales (CFTC, CFDT, CFE-CGC, CGT, FO, FSU, Solidaires, UNSA $\left.{ }^{(4)}\right)$. Cependant, faute de stratégies claires et de perspectives données à ces journées de mobilisation, le mouvement s'essouffle. Au sein de syndicats traditionnellement contestataires, comme la CGT ou Sud, une partie de la base s'interroge sur la volonté réelle de leurs confédérations de maintenir un rapport de force qui commence à se construire par l'action de rue. Le bilan controversé de cette mobilisation discontinue conduit à s'interroger sur les conséquences qu'a pour l'action

(2) Cette crise qui va conduire à la plus importante récession économique depuis la grande dépression des années 1930 démarre aux Etats-Unis en 2007 par l'éclatement de la bulle immobilière (crise des subprimes). Elle se poursuit à l'automne 2008 par un krach boursier et la faillite de plusieurs établissements financiers, dont la banque d'investissement Lehman Brothers. Cette crise bancaire, qui touche bientôt le reste de la planète et particulièrement l'Europe, engage les gouvernements européens et la Banque centrale européenne (BCE) à intervenir massivement en injectant des fonds pour sauver le système bancaire.

(3) Goarant C., Minni C. (coord.), Pasquereau A., Rémy V., Tovar M.-L., Debauche E., Thélot H. (2010), «Emploi, chômage, population active : bilan de l'année $2009 »$, Dares Analyses, $\mathrm{n}^{\circ}$ 50, juillet.

(4) Confédération française des travailleurs chrétiens; Confédération française démocratique du travail; Confédération française de l'encadrement - Confédération générale des cadres; Confédération générale du travail; Force ouvrière; Fédération syndicale unitaire; Union syndicale solidaires; Union nationale des syndicats autonomes. syndicale la progressive préférence pour la négociation d'entreprise et le «dialogue social» (BÉROUD, Yon, 2009). Le conflit qui se déroule à cette période à l'usine Continental de Clairoix apparaît en tout point à rebours d'une stratégie syndicale réformiste. Dès les premiers jours, le mouvement s'affranchit de la tutelle des institutions syndicales et se dote d'un comité de lutte, instance chargée de débattre des moyens donnés à un mouvement se présentant comme l'expression concrète d'antagonismes de classe. Faut-il alors y voir l'expression par les ouvriers d'une critique "par le bas» des confédérations ?

Pour comprendre la radicalisation de ce mouvement social, nous avançons dans cet article qu'il faut surtout la réinscrire dans son contexte sociopolitique local et sa temporalité, afin de saisir les relations entre les salariés, leurs représentants et la direction. Ce conflit émerge dans l'une des premières entreprises à avoir mis en place les accords de retour aux quarante heures en 2007, dits « travailler plus pour gagner plus », signés ici par un syndicat CFTC majoritaire et la CFE-CGC. Il s'inscrit également dans un bassin industriel marqué par des décennies de restructurations, avec un possible transfert de «capital militant» (Matonti, Poupeau, 2004) et de savoirs sur les stratégies de lutte entre usines. Dans un premier temps, nous mettrons en exergue les conditions locales de cette mobilisation marquée par des dissensions syndicales et des conflits sociaux antérieurs, qui contredisent l'idée d'une pacification des relations ouvrières. Nous poursuivrons par l'étude des acteurs, internes et externes à l'usine, qui ont infléchi la contestation sociale en lui donnant une portée plus générale et politique de «critique sociale» (voir encadré 1): cette radicalisation a été portée par la majorité des salariés, même ceux éloignés des organisations syndicales, qui partageaient les mêmes sentiments $\mathrm{d}$ 'humiliation et de colère. Dans un troisième temps, en nous inspirant de la notion d'économie morale d'Edward P. Thompson (1988a), nous examinerons les ressorts subjectifs de cette mobilisation collective qui reposent sur un double registre, matériel et symbolique, de la «réparation»: compensation financière de la perte d'emploi et du salaire, mais bien au-delà, réparation morale qui impose la reconnaissance sociale, publique, de la légitimité de la lutte.

\section{Un symbole de la "casse sociale"}

Implanté à Clairoix, dans l'Oise, le site de l'usine Continental abrite durant un siècle une filature de soie avant de se reconvertir dans la fabrication de chambres à air et de pneumatiques en 1936, à la suite de son rachat par la société Englebert. 


\section{Encadré 1 \\ Méthodologie d'une enquête encore en cours}

L'article s'appuie sur une enquête sociologique menée depuis avril 2009, quelques semaines après le début du conflit social. Elle s'est largement appuyée sur l'observation directe qui a suivi le calendrier du mouvement : assemblées générales sur le parking de l'usine, nombreux échanges et discussions à cette occasion avec les salariés de Continental, participation à plusieurs manifestations (Amiens, Beauvais, Compiègne, Paris, Sarreguemines), aux meetings organisés devant l'usine mais aussi à Compiègne et à Amiens, concernant le procès des sept salariés poursuivis pour les destructions commises à la sous-préfecture de Compiègne et les audiences où comparaissait Xavier Mathieu, un des délégués de la CGT, pour avoir refusé le prélèvement de son ADN par la police. Nous sommes ainsi entrés progressivement en relation avec des anciens salariés et tous les responsables syndicaux, quelle que soit leur affiliation. La permanence de ces contacts, qui ont survécu à la liquidation de l'usine, nous a permis ensuite de réaliser une enquête quantitative (janvier 2013), à partir des listes d'adresses des anciens salariés (1100 au 31 décembre 2009) détenues par le comité d'entreprise. Sa finalité est d'objectiver certaines réalités du reclassement des anciens salariés. Des entretiens ont ensuite commencé auprès des personnes qui, dans le questionnaire (390 ont été retournés (1)), ont donné leur accord pour nous rencontrer (soit une centaine d'ex-salariés de Continental). Ce travail de recherche s'inscrit donc dans la longue durée, temps ici nécessaire pour créer une relation de confiance entre les enquêteurs et les enquêtés.

Les modalités de l'enquête ont été contraintes par la nature particulière des événements observés, qui, dans un premier temps, rendait l'entretien formel inenvisageable. Certains matériaux sont encore à l'état brut ou demandent des recoupements et ne seront donc pas traités ici (essentiellement les données ethnographiques). L'article oriente essentiellement le propos sur un contexte local de lutte qui est reconstruit, à froid, à partir des premiers entretiens réalisés entre octobre 2013 et janvier 2014 avec des élus syndicaux ayant participé au mouvement. Certains ont siégé au comité d'entreprise (CE) qui a été dissous définitivement fin février 2014. Quoique les principaux protagonistes du mouvement nous accordent leur confiance, quoique nos identités soient bien connues, certains salariés, très actifs dans le Comité de lutte, ont pour l'instant esquivé tout entretien, au premier chef Xavier Mathieu. Notre corpus se trouve donc à ce jour épars, non clos et informé par les différents entretiens qui ont pu aboutir. Compte tenu de son hétérogénéité actuelle, nous avons décidé de nourrir le propos de cet article des significations que les militants du Comité de lutte, constitué de syndicalistes CGT ou FO, ont donné à ce mouvement. II laisse dans l'ombre d'autres points de vue attachés aux positions et rôles investis par certains adhérents et représentants de la CFTC, de la CFDT et de la CFE-CGC.

Les entretiens utilisés ici ont été réalisés avec des membres du Comité de lutte (Michel Bonte et François Lefèvre de FO, Jacques Pichon et Patrick Lachambre de la CGT), un responsable de la CFDT (Julien Robin), de la CFTC (José Munes) et un ancien ouvrier (Robert Jaquin), qui a participé à ce mouvement social sans avoir pris part au Comité de lutte. Afin de préserver leur anonymat, nous leur avons donné un prénom et un nom fictifs. Nous avons utilisé de nombreux documents et articles de presse qui donnent des traces factuelles. En même temps, nous nous appuyons sur une recherche précédente réalisée dans le cadre d'une thèse. Elle a conduit l'un d'entre nous à rencontrer des salariés de l'entreprise Chausson, qui ont mené un long conflit social contre la fermeture de leur entreprise au milieu des années 1990. Le leader de ce mouvement, Roland Szpirko, réapparaît dans le conflit des Conti (Depoorter, 2006).

(1) Un traitement qualitatif des commentaires libres ayant accompagné les réponses au questionnaire est proposé dans l'article.

L'usine prend rapidement de l'essor et devient une entreprise majeure du Compiégnois ${ }^{(5)}$, comptant 1500 salariés au début des années 1960 (LAZZAROtTI, 1968, p. 327). Intégrée au groupe Uniroyal à la fin des années 1950, elle passe sous le contrôle de l'entreprise allemande Continental en 1979 , au moment de la reprise de la totalité de l'activité pneumatique européenne du fabricant

(5) Troisième pôle industriel de Picardie, le Compiégnois compte plus d'un quart d'emplois industriels, soit trois points au-dessus de la moyenne d'une région pourtant placée au troisième rang régional pour l'emploi dans l'industrie. Avec, à l'époque, trois grandes usines de fabrication de pneumatiques (Continental, Dunlop et Goodyear qui vient également de fermer), la Picardie était, pour l'emploi dans ce secteur, la seconde région française, derrière l'Auvergne. Pour plus de détails, voir Insee Picardie (2006), «Compiègne: une zone d'emploi urbaine très industrialisée», in L'industrie dans les territoires picards, novembre, pp. 67-74. américain. À la fin de l'année 2008, l'usine de Clairoix emploie 1125 salariés dont 922 ouvriers $(82 \%)$ pour 132 Etam (Employés, techniciens et agents de maîtrise) et 71 cadres ${ }^{(6)}$. Malgré la tendance à une réduction des effectifs liée à la modernisation des outils de production, les recrutements sont nombreux durant les dernières années. Les chiffres du bilan social montrent que $22 \%$ des salariés sont présents dans l'entreprise depuis moins de six ans alors que la moitié des personnels a plus de quinze ans d'ancienneté (7). Nous retrouvons ces caractéristiques dans l'enquête par questionnaires

(6) Bilan social d'établissement, 2007-2008-2009.

(7) $40 \%$ des salariés de l'usine sont alors âgés de moins de 40 ans. Dans cette usine dont l'activité nécessitait beaucoup de travaux de manutention, les hommes étaient fortement majoritaires. Ils représentent alors $94 \%$ de l'effectif total (et $96 \%$ des ouvriers). 
réalisée en janvier 2013 qui, bien que non représentative de l'ensemble des salariés, permet de préciser l'analyse. Si un tiers des salariés répondants habite Compiègne ou les villages voisins de Clairoix, un second tiers est domicilié dans les communes plus éloignées (entre 10 et 20 kilomètres). Enfin, un troisième tiers demeure à plus de 30 , voire 40 kilomètres de l'usine. Autrement dit, la zone de recrutement des salariés allait au-delà de l'Oise pour atteindre les départements de la Somme et de l'Aisne. L'enquête donne à voir une classe ouvrière à la fois ancrée dans le monde rural et installée dans une histoire industrielle depuis plusieurs générations. Cette représentation est confortée par le faible taux de travailleurs étrangers ( $4 \%$ de l'effectif), bien en dessous des moyennes observables dans les usines $\mathrm{du}$ sud de l'Oise, comme Chausson ou d'autres entreprises du bassin creillois voisin. Dans quel contexte la fermeture de cette usine intervient-elle ?

\section{“Travailler plus pour gagner plus!"}

L'annonce est faite très tôt le matin du 11 mars 2009, par voie de presse. Elle est confirmée aux salariés vers 8 heures par «un grand directeur venu d'Allemagne», nous dit Robert Jaquin, 56 ans, ouvrier pendant trente ans, se souvenant encore aujourd'hui (l'entretien a été réalisé en janvier 2014) des conditions dans lesquelles il apprend la nouvelle. "Je me rappelle comme si c'était hier. On nous réunit dans un grand entrepôt pour nous dire que la boîte elle ferme parce qu'on est trop cher... comme cela du jour au lendemain! [...] nous réunir comme cela pour nous dire ça comme cela! Vous auriez $v u$ la tête des gens qui pleuraient, qui sortaient pour aller dans leur vestiaire... ça nous a fait tout drôle!» Cette annonce provoque un effet de sidération. L'information passe en boucle sur les ondes des radios. Très vite, le travail est arrêté. Des salariés se rassemblent devant les portes de l'usine. Ils sont bientôt rejoints par les médias qui vont couvrir quasi quotidiennement cet événement jusqu'à la signature des accords entérinant le plan de sauvegarde de l'emploi. Les conditions de la fermeture font vivement réagir les ouvriers de Clairoix, qui l'interprètent comme une véritable trahison et y décèlent un grand mépris de la direction à leur égard : «il y avait eu beaucoup de sacrifices avant la fermeture», nous dira Jacques Pichon, ouvrier, délégué CGT. Il fait ici référence à l'accord organisant le retour aux quarante heures de travail hebdomadaire et en vertu duquel la direction s'était engagée à maintenir la production sur le site jusqu'en 2012.

Après plusieurs mois de négociations, l'accord de retour aux quarante heures avait été signé en septembre 2007 par la CFTC, le syndicat majoritaire, et la CFE-CGC. Cet accord était mal passé dans les ateliers et avait fait l'objet de vives contestations de la part de la CFDT et de la CGT. Néanmoins, aucun mouvement de grève ne s'était déclenché. Les relations sociales dans l'entreprise étaient-elles donc apaisées depuis ce moment où, agissant en «syndicat responsable», le syndicat chrétien parvint, en 2004, à remporter les élections professionnelles ? Cette année-là, il obtint $43 \%$ des voix aux élections du comité d'entreprise devant la CGT (27\%) et la CFDT $(26 \%)$ et ravit à cette dernière le poste de secrétaire grâce à une alliance avec la CFE-CGC. La CGT, longtemps majoritaire, réussit à garder deux délégués. Cette redistribution des mandats était inscrite dans l'histoire syndicale de l'entreprise, qui avait vu la section CGT perdre progressivement sa position dominante au milieu des années 1990, à la suite du départ d'une partie de ses membres à Force ouvrière et du licenciement d'un délégué, Jean-Marc Iskin, qui fut un des animateurs d'une grande grève en 1994. Les entretiens menés auprès des différents délégués syndicaux, quelle que soit leur affiliation, désignent les événements qui entourèrent cette grève de 1994 comme le moteur du basculement des forces syndicales. Elle constitue un tournant dans l'histoire de cette usine où le modèle de "dialogue social» voulu par la direction s'était imposé par le truchement d'un syndicalisme réformiste. Il s'est accompagné d'un «chantage à l'emploi» et de sanctions pour produire du consentement, voire d'une certaine violence physique assumée par cette direction (voir encadré 2).

En 2007, la CFTC a donc la main pour engager des négociations avec la direction concernant un accord de quarante heures. Celui-ci est mis en œuvre dans un contexte où, depuis des années, l'épouvantail de la fermeture est constamment agité par le groupe Continental, qui place ses sites en concurrence et en lutte permanentes pour leur survie - une méthode que le cabinet chargé de l'expertise économique pour le comité d'entreprise qualifiera de «véritable darwinisme industriel ${ }^{(8)} 》$. Soumis à la pression de la direction, le responsable de la CFTC négocie ce qu'il considère être un bon accord pour la pérennisation du site :

"Si je signe un accord, je m'engage à quelque chose. Si je mets une durée de deux ans, qu'est-ce que je vais gagner dans l'histoire en deux ans? Je n'ai pas voulu mettre de date. J'ai voulu mettre le mot "pour la pérennité du site et des emplois". C'est tel quel. Ça me paraissait moi, plus sensé et beaucoup plus porteur que de dire, on va faire un accord pour deux ans et je vous garantis l'emploi pour deux ans. Mais bien sûr cet accord, je l'ai refusé pendant un an! J'étais majoritaire, j'avais ce pouvoir de dire non pendant un an. [...] Je m'y oppose. Travail gratuit, je dis non! J'ai dit non et la menace de la fermeture était sur mon dos à moi! La direction me pointait du doigt en disant: "Il ne veut pas, il ne veut pas." J'ai dit : "La CFTC dit non, mais propose." [...] On a signé à une condition: vous m'embauchez les 130 intérimaires, vous donnez $100 €$ par mois aux salariés pour

(8) Rapport Secafi, décembre 2010 portant sur l'analyse de l'exercice 2009. A priori non public, il nous a été présenté lors d'une entrevue avec un membre du CE. 


\section{Encadré 2}

\section{La grève de 1994}

Le licenciement de Jean-Marc Iskin, responsable syndical CGT et militant de Lutte ouvrière, intervient en 2002 au terme de huit années de procédure judiciaire. Une décision du Conseil d'État vient alors clore une affaire qui a pour origine le déclenchement d'un conflit social en juin 1994. Revendiquant une augmentation des salaires et l'embauche en contrat à durée indéterminée d'intérimaires, les ouvriers bloquent l'usine durant une dizaine de jours. Après cet épisode, dix-neuf personnes sont licenciées, puis réintégrées quelques mois plus tard, à l'exception du militant trotskiste. La direction lui reproche d'avoir «réitéré des appels au blocage de l'usine, empêchant ainsi la libre circulation des marchandises, même après que les salariés aient décidé la reprise du travail(1) ». Si, dans un premier temps, l'inspection du travail refuse le licenciement, sa hiérarchie le valide. La décision du ministère est ensuite cassée par le tribunal administratif. Le militant de Lutte ouvrière, ouvrier professionnel, embauché au milieu des années 1980, est finalement exclu en 2002.

Mais c'est surtout un autre épisode, survenu durant ce conflit social, qui reste gravé dans les mémoires : l'intervention de vigiles sur le piquet de grève, affaire dont la presse nationale s'était fait l'écho (2). Les ouvriers décrivent une véritable «action commando» qui se déroule le 30 juin, vers 3 heures du matin, précise Patrick Lachambre (ouvrier, délégué syndical CGT à l'époque). Elle était manifestement bien préparée. Les vigiles avaient pris soin de couper les lignes téléphoniques avant de foncer sur le petit groupe d'ouvriers. Leur brutalité obligea quelques grévistes à fuir et à se jeter dans l'Oise, qui longe le parking de l'usine. D'autres eurent moins de chance et furent durement molestés, comme le rappelle Patrick Lachambre en janvier 2014 : "Le patron les a fait monter dans le camion et quand ils sont arrivés devant le portail, il y avait très peu de monde dehors. Les mecs sont sortis et ont tabassé, les copains se sont foutu la gueule dans l'eau parce qu'ils ne voulaient pas prendre des coups de matraque. Le local syndical a été broyé. J'ai un copain qui s'est fait tapé dessus, on l'a fait hospitaliser, il a eu la rate éclatée. »

Le directeur de l'époque est finalement reconnu responsable de cette action violente qui, à sa façon, donne une idée de la nature des relations sociales de l'époque. Le tribunal de grande instance de Compiègne le condamne à une peine de quatre mois de prison avec sursis. À la suite de cette grève, le groupe Continental décide de baisser la production de l'usine de Clairoix, de la placer sous surveillance pendant une année et annonce un plan de licenciements de 149 personnes. La violence qui s'est exercée lors de ce mouvement de grève, les démêlés judiciaires qui ont suivi et les sanctions infligées par le groupe allemand ont contribué à modifier durablement les relations sociales et les alliances syndicales et, très certainement, pour la frange la plus à gauche de la CGT, à nourrir un sentiment d'injustice.

(1) Conseil d'État, décision du 26 juin 2002, n 219940.

(2) Libération, 23 février 1995.

deux heures et demie de travail en plus par semaine, dont une heure rémunérée non imposable... C'était au moment de cette loi de Sarkozy, "Travailler plus pour gagner plus”, vous savez... On a été la première entreprise à l'appliquer."

(Entretien avec José Munes, ouvrier, représentant CFTC, réalisé en janvier 2014.)

La fabrique du consentement (Burawoy, 2011) reposait ainsi, depuis des années, sur les compensations financières de la pénibilité du travail mais également sur les possibilités de faire carrière dans l'usine (9) et sur la sécurité de l'emploi qu'incarnait la puissance du groupe allemand. L'entreprise

(9) Notre enquête sur le reclassement montre que la quasitotalité des agents de maîtrise de la production était d'anciens ouvriers. Ils ont bénéficié des conditions «d'employabilité» (Boltanski, Chiapello, 1999) ordinairement mises en œuvre dans les phases de pérennité économique. Les possibilités d'évolution professionnelle et de carrière s'inscrivaient ainsi dans des processus de coproduction collective (ZIMMERMANN, 2011) entre employeur et salariés et ces derniers y formaient également le projet d'assurer l'avenir de leurs enfants (LINHART, 2005). Les transformations productives en cours depuis la fin des années 1980 sont venues fragiliser ces formes de valorisation sociale qui semblaient acquises pour les familles ouvrières (Beaud, Pialoux, 1999). continuait à recruter, ce que l'accord de 2007 programmait, de même qu'il prévoyait des investissements devant assurer la pérennité du site. Ainsi, après une période probatoire, les fils des salariés pouvaient espérer bénéficier d'une priorité à l'embauche.

«On gagnait bien quand même. Je vois, en étant toujours du matin, sans faire de nuit, je gagnais $2100 €$. C'est quand même pas mal en faisant quarante heures. On était arrivé à avoir beaucoup de primes. On travaillait dans les produits quand même où il y avait beaucoup de séquelles, on acceptait... On avait une prime de chaleur au-dessus de $30^{\circ}$; quand tu travaillais le dimanche, tu avais une journée de récupération.»

(Entretien avec Patrick Lachambre, militant CGT, réalisé en janvier 2014.)

Le compromis salarial qu'incarnait à sa manière la CFTC s'établissait à la fois sur des contreparties matérielles et un contrat moral sur l'avenir du site. Néanmoins, l'accord des quarante heures cristallisa plus de tensions et de divisions syndicales qu'il n'y parut d'abord. Il ne fit pas l'unanimité. Le délégué CFTC le reconnaît lui-même : «On m'a dit que je cédais à la direction, mais non, non, on gagnait sur 
tout [...]. C'était un super accord. D'ailleurs, il n'y a pas eu de grèves... même les gens de la CFDT ont suivi...» De son côté, la CFDT, selon les propos que nous avons recueillis auprès de l'un de ses représentants de l'époque, disait le contraire : «Ni la CFDT ni la CGT n'étaient d'accord... Quel était le gain ? Le seul gain, on travaillait le week-end et les jours fériés... J'ai toujours dit qu'on perdait en fait...» (entretien avec Julien Robin en janvier 2014). Cet accord a alimenté les contentieux qui sont allés grandissants entre courants syndicaux. Ils ont ressurgi avec force lors de la mobilisation de 2009, contribuant à consommer les fractures entre CGT et CFTC, puis entre CGT et CFDT.

\section{“Une stratégie pour faire fermer la boîte!"}

La position de la CFTC locale était que la crise de 2009 était passagère car, précise José Munes, pour cette usine française, "le carnet de commandes de l'entreprise était plein». Le but du groupe Continental, poursuit-il, était certes de fermer à moyen terme le site de Clairoix : "On le savait, c'était une menace depuis 1994.» Mais, selon lui, l'usine n'aurait pas fermé aussi rapidement si le conflit avait su trouver dans la négociation d'entreprise et dans le Code du travail les ressorts pour entamer des procédures administratives et faire les recours qui s'imposaient. La CFDT locale, quant à elle, s'engagea dans une autre voie. Elle n'eut de cesse de lancer des signaux d'alerte dès qu'elle percevait des indices tangibles d'une restructuration à venir, réclamant des réponses de la part de la direction. Ses délégués provoquèrent notamment, en fin d'année 2008, une réunion extraordinaire du comité d'entreprise, sans toutefois obtenir la moindre information de la part de leur employeur. En novembre, l'un d'entre eux, nous dit Julien Robin, délégué syndical CFDT, avait en effet reçu un courrier anonyme, envoyé sans doute par un cadre du site, dans lequel se trouvait un tableau de bord datant de janvier 2008. Ce document, qui fut publié par la presse locale dès le début du conflit ${ }^{(10)}$, prévoyait l'augmentation progressive des coûts de production de l'usine de Clairoix. Très vite, pour ce responsable syndical, l'explication lui sembla évidente. L'accord sur les quarante heures s'inscrivait dans une "stratégie [qui] avait été faite pour fermer le site. La direction a commencé à investir, à en faire une vitrine. On était la vitrine de Continental. On avait les meilleurs outils, on avait la meilleure amélioration de production [...]; sur le coup tu te dis, ben ça a l'air de bien se dérouler ». Au service des méthodes où il était technicien, Julien Robin observait néanmoins que, parallèlement, rien n'était fait pour endiguer la dégradation des coûts de production, bien au contraire : «En deux ans, on a détérioré tous les coûts [...]. Ils [la

(10) Le Courrier picard, 13 mars 2009. direction] sont venus pour fermer l'usine [...]. Le matériel, l'outil a été détérioré, sciemment. On ne réparait pas. Les absences n'étaient pas remplacées, les congés non plus...» Bien plus, selon lui, les remaniements successifs de postes à la direction de l'entreprise étaient programmés : "C C'était organisé. La direction a reçu des ordres de Hanovre. Ça a duré un an et demi... Les accords n'ont fait qu'aggraver le coût horaire... La CFTC n'y croyait pas... il faut dire que la direction la tenait un peu en otage en lui rappelant qu'elle avait signé les quarante heures...» Ce délégué CFDT avertit les autres syndicats et les élus qui, bientôt, partagèrent son analyse : "Tout cela, nous dit Michel Bonte, ouvrier, délégué $\mathrm{FO}$, lors d'un entretien réalisé en octobre 2013, c'était orchestré, organisé de longue date, tout cela était préparé et ils ont profité de la crise pour s'engouffrer dans la brèche... ils se sont dit, c'est le moment ou jamais de se débarrasser d'un site, c'est le moment.»

Les expertises commanditées par le comité d'entreprise confirment les soupçons des responsables syndicaux CFDT, CGT et FO : la moindre profitabilité du site a été méticuleusement organisée pour justifier la fermeture(11). Devant le mutisme de la direction, les syndicats lancent une procédure d'alerte et attendent la réunion du comité central d'entreprise du 16 mars 2009 à Reims. Dès les premiers jours de mars, «des rumeurs», relayées par la presse spécialisée ${ }^{(12)}$, se font plus insistantes sans, pour autant, être confirmées par la direction. Au contraire, celle-ci apporte même un démenti dans un bulletin d'information adressé le 5 mars à l'ensemble des personnels. "Ils nous ont menti jusqu'au dernier moment », nous dit Jacques Pichon, délégué CGT. Cette stratégie de la direction est vue comme un acte ultime de forfaiture qui a contribué, d'après les militants CGT, à alimenter la défiance envers la parole patronale, à arc-bouter les positions et à radicaliser le conflit. Le syndicat majoritaire (CFTC) se retrouve alors isolé dans ses positions. Jusqu'au bout, son représentant élu s'emploie à rassurer les salariés, garantissant que la direction ne peut se dédire de son «contrat moral» :

«La CFDT dit quelque chose dans le journal, la CGT dit la même chose, la CFE-CGC commence à percuter et lui [le responsable de la CFTC], à chaque fois que je fais une intervention pour dire que ça ferme, il tient avec la direction [...]. Jusqu'au bout du bout, la direction lui a fait comprendre que ce n'était qu'une rumeur. Mais quand il est alerté, comme moi à 6 heures du matin que Continental faisait une réunion extraordinaire à 8 h 30 pour l'ouverture des livres 3 et $4^{(13)}$, il est devenu fou. Il est obligé de se rendre

(11) Rapport Secafi, décembre 2009.

(12) «Menace de fermeture de l'usine Continental de Clairoix », L'Usine nouvelle, 2 mars 2009.

(13) C'est-à-dire l'ouverture de la procédure d'informationconsultation dans le cadre de la mise en œuvre du plan de sauvegarde de l'emploi (PSE). 
compte que la direction ment sur les chiffres, ment sur la stratégie.»

(Entretien avec Julien Robin, délégué CFDT, réalisé en janvier 2014.)

La médiatisation des premiers jours du conflit participe à en faire un problème politique. Il devient alors le symbole d'une «casse sociale» menée par une entreprise peu scrupuleuse, trahissant ses engagements à l'égard de ses salariés qui avaient pourtant accepté de revenir sur des acquis sociaux pour accroître la compétitivité de leur site de production. Les réactions consternées de l'ensemble des élus locaux, quelle que soit leur appartenance politique, et du gouvernement ne se font pas attendre. Son porte-parole, Luc Chatel, secrétaire d'État auprès de la ministre de l'Économie, chargé notamment de l'Industrie, qualifie même cette décision de «trahison ${ }^{(14)}$ », précisant «que si, le groupe persistait dans sa volonté d'une restructuration du site de Clairoix, il aurait à justifier devant les tribunaux de la motivation de tels licenciements ». Le ministre de l'Emploi s'était déjà exprimé sur le sujet, le 11 mars matin, rappelant les obligations de la firme allemande en matière d'information des salariés ${ }^{(15)}$. L'affaire prend aussi une dimension internationale quand, au cours de la même journée, le président de la République, en déplacement à Berlin, intervient publiquement, interpellant directement la Chancelière. Devant ce concert d'indignations, y compris au plus haut sommet de l'État, les salariés, délégués syndicaux CGT et FO en tête, se mobilisent, organisent plusieurs rassemblements, en appellent aux élus et responsables politiques alors que, parallèlement, la direction confirme dans la presse le caractère irrévocable de sa décision ${ }^{(16)}$.

La perspective de la fermeture a un tel impact que les élus locaux de tous bords ouvrent les portes de leurs bureaux. Les élus UMP apportent publiquement leur soutien aux salariés, comme ici, Michel Gonnot, député de la circonscription : «D'abord, je suis totalement solidaire des salariés de l'entreprise et de leurs représentants dont la direction se moque depuis des mois $[\ldots]$; je reste persuadé, qu'au-delà de la crise de l'automobile qu'il subit mais dont il se sert aussi, le groupe Continental a un sinistre projet à Clairoix qu'il va falloir intelligemment contrecarrer au plan local ${ }^{(17)}$.» Les responsables syndicaux de l'usine sont entendus par Claude Gewerc, socialiste, président du conseil régional de Picardie, reçus par Philippe Marini, sénateur maire UMP de Compiègne et bientôt par Raymond Soubie, conseiller social du président de la République, en marge de la manifestation parisienne du 25 mars. Ils demandent à l'État de s'impliquer dans le dossier en provoquant une réunion tripartite avec la direction de Continental. Durant ce mois de mars émaillé de manifestations, ceux que les médias commencent à appeler les Conti mesurent le vaste élan de sympathie qu'ils suscitent dans la population du Compiégnois et bien au-delà. C'est dans cette période que le mouvement se construit, consolidant ses raisons d'être. Il affine aussi ses moyens d'expression et ses modes de représentation. Deux noms sont bientôt associés au mouvement : Xavier Mathieu, qui devient la figure emblématique du Conti contestataire, et Roland Szpirko, leader du mouvement social qui eut lieu chez Chausson, appelé en renfort. Ce dernier, perçu comme un personnage un peu énigmatique, du fait de son engagement à Lutte ouvrière (LO, dont il ne fait pas mystère), a tenu un rôle important dans la construction et la politisation du mouvement, notamment dans la constitution d'un comité de lutte. Cette alliance de la CGT et de LO peut contribuer à expliquer comment le conflit social a pu changer de registre, passant d'un conflit du travail à une lutte politique; néanmoins elle n'explique pas les conditions sociales de cette rencontre et de l'adhésion de la majorité des salariés. C'est ce que nous allons maintenant analyser.

\section{Dans l'arène politique}

Très rapidement, le conflit social (voir encadré 3) entre pleinement dans l'arène politique et fait craqueler le vernis de l'apparent consensus. Le rôle de la presse dans la couverture des faits a sans doute contribué à lui donner une ampleur certaine mais, de façon réciproque, le mouvement des Conti est un exemple de la façon dont les ouvriers se sont appropriés avec habileté les codes sociaux de l'univers médiatique, réussissant à le mobiliser en permanence autour d'actions revendicatives ou de meetings de soutien à leur cause. L'utilisation qu'ils ont faite des médias est révélatrice d'une stratégie consistant à vouloir agir directement dans le champ politique (ChAmpagne, 1984), avec pour objectif d'engager l'État à entrer dans le jeu des négociations avec leur direction.

(14) «Lagarde choquée par la fermeture de l'usine Continental à Clairoix », L'Express.fr, 13 mars 2009.

(15) «Clairoix (60). Colère chez Continental et ses 1120 salariés sur le carreau», Le Courrier picard, 12 mars 2009.

(16) "Continental "ne reviendra pas" sur sa décision», Le Courrier picard, 16 mars 2009.

(17) «Continental: le sinistre projet», billet publié sur le blog de Michel Gonnot, député UMP de la circonscription Compiègne-Nord; en ligne à l'adresse : http://gonnot.overblog.com/article-28657369.html; consulté le 25 mars 2014. 


\section{Encadré 3}

\section{Chronologie des principaux événements}

11 mars 2009 : Continental annonce la fermeture de deux sites de production : Clairoix dans l'Oise et Stöcken près de Hanovre en Allemagne. Le site de Clairoix fermerait le 31 mars 2010 à la suite de deux plans de licenciement (octobre 2009 et mars 2010).

12 mars 2009 : Réunis en assemblée générale, les salariés décident la grève. Le site est bloqué à 20 heures.

13 mars 2009 : Publication dans la presse locale (Courrier picard) du document attestant la programmation de la «dégradation » des coûts de production qui devait justifier la fermeture de l'usine de Clairoix. Une délégation formée de responsables syndicaux est reçue au siège du conseil régional.

16 mars 2009 : Près de 800 salariés manifestent à Reims devant les locaux où se tient la réunion du comité d'entreprise.

19 mars 2009 : Journée de mobilisation nationale. La manifestation des Conti, partie de Clairoix, rejoint le cortège compiégnois. Plus de 10000 personnes manifestent dans la cité impériale.

23 mars 2009 : Reprise du travail après onze jours de grève.

25 mars 2009 : 700 Conti (18 cars) partent manifester à Paris. Une délégation est reçue par Raymond Soubie, conseiller du président de la République.

31 mars 2009 : Une audience en référé visant à annuler le plan de sauvegarde de l'emploi (PSE) se tient au tribunal de Sarreguemines (siège social de Continental France). Elle est demandée par les organisations syndicales, qui, par ailleurs, boycottent la réunion du comité central d'entreprise (CCE) à Nice où la direction doit présenter les éléments du plan social. Celui-ci prévoit une indemnité d'un sixième par année d'ancienneté, sans toutefois pouvoir excéder au total vingt mois de salaire pour les plus anciens, et un congé de mobilité de cinq mois. Les organisations syndicales demandent une prime de 200000 euros et le maintien de leur contrat de travail jusqu'en 2012.

16 avril 2009 : Le secrétaire du CE est assigné en référé devant le tribunal de Compiègne pour avoir refusé de signer la convocation au comité d'entreprise du 20 avril au cours duquel la direction informe les représentants des salariés de son intention de retirer les moules servant à la fabrication des pneus.

21 avril 2009 : Le tribunal de Sarreguemines déboute la demande d'annulation de la procédure de fermeture de l'usine. Les salariés qui étaient à la sous-préfecture de Compiègne "saccagent» des bureaux et du matériel informatique. Le même jour, le local de sécurité de l'usine est rendu inutilisable.

22 avril 2009 : L'usine de Clairoix est totalement à l'arrêt, les salariés sont placés en chômage technique.

23 avril 2009 : Plus d'un millier de salariés et leurs familles partent à Hanovre en train depuis Compiègne pour manifester devant l'assemblée générale des actionnaires. Ils sont accueillis à la gare par 1500 salariés allemands du groupe Continental AG. Le même jour, le gouvernement annonce un projet de reprise du site par une société de Dubaï (MAG). Ce projet n'aboutit pas, Continental lui adressant une fin de non-recevoir.

27 avril 2009 : 500 Conti se rendent à Creil pour soutenir les sept salariés convoqués par le SRPJ (service régional de police judiciaire) à la suite des dégradations commises à la sous-préfecture de Compiègne. Le même jour, sur son blog, le député François-Michel Gonnot affirme, concernant la destruction du poste de garde que «les casseurs voulaient rendre - et ont rendu - l'ensemble de l'usine inutilisable. [...] Les casseurs, une bande de salariés instrumentalisés par les trotskistes de Lutte ouvrière ont mené ces opérations [...] de façon précise et organisée ». Xavier Mathieu lui répond : «Si François-Michel Gonnot a l'habitude de côtoyer des moutons dans son monde, qu'il sache que ce n'est pas le cas à Clairoix. Personne n'est instrumentalisé, ni manipulé. ”

29 avril 2009 : Première réunion «tripartite» à Bercy et manifestation des Conti pour soutenir leurs représentants. Le responsable de la CFDT déclare à la presse (Courrier picard) : «Nous venons avec des revendications claires. Nous souhaitons un plan de reconversion de 48 mois, soit jusqu'en 2012, ainsi qu'un plan de départs en préretraite à 52 ans pour tous les volontaires. Enfin, nous demandons une somme de $200000 €$ par ouvrier en compensation du préjudice moral subi. "

$1^{\mathrm{er}}$ mai 2009 : Les Conti défilent à l'occasion de la fête du travail à Compiègne où le rassemblement est massif (plusieurs milliers de personnes). Une manifestation festive se déroule l'après-midi à Thiécourt.

6 mai 2009 : 300 à 400 salariés de Clairoix occupent l'usine de Sarreguemines afin d'obtenir un rendez-vous avec la direction allemande. Lors de l'assemblée générale du lendemain, le lancement de quatre procédures émanant de l'intersyndicale, du comité d'entreprise et du comité central d'établissement est annoncé.

12 mai 2009 : Le Courrier picard publie les premiers éléments du rapport du cabinet d'expertise, Secafi, mandaté par le comité d'entreprise. Les experts réfutent l'argument du manque de compétitivité du site pour justifier la fermeture, qu'ils expliquent plutôt par une «délocalisation rampante» de la production vers les pays à bas coût. La direction annonce le report d'un mois de la mise en œuvre du PSE, se donnant plus de temps pour négocier les mesures d'accompagnement social des licenciements. En contrepartie, les syndicats s'engagent à retirer les procédures judiciaires en cours et acceptent une reprise du travail prévue le 2 juin après la remise en état du local de garde.

14 mai 2009 : Réunion du comité central d'entreprise à Nice.

18 mai 2009 : 600 salariés de Continental manifestent devant la Bourse de Paris. 
27 mai 2009 : Plusieurs centaines de salariés de Continental manifestent devant le siège du Medef (Mouvement des entreprises de France) à Paris.

29 mai 2009 : Accord entre la direction et les représentants des salariés sur le contenu du plan social qui prévoit une prime extra-légale de $50000 €$ pour tous les salariés, quelle que soit leur ancienneté, en sus des indemnités légales de licenciement, et un congé de mobilité de 24 mois.

30 mai 2009 : L’assemblée générale réunie devant l'usine accepte les accords.

1er janvier 2010 : L'usine Continental de Clairoix est officiellement fermée.

\section{La radicalisation}

Les premiers signes d'une radicalisation du mouvement se manifestent lors du déplacement, le 16 mars 2009, de la maire de Beauvais, future tête de liste aux élections régionales, accompagnée de sept autres élus de 1'UMP. Malmenés par les ouvriers qui reprochent au gouvernement dirigé par la droite de n'avoir pas tenu ses promesses de campagne, ils quittent le site prématurément, ce dont la presse locale se fait l'écho ${ }^{(18)}$. Mais le clivage politique est encore plus net après les incidents survenus le 21 avril 2009 à la sous-préfecture de Compiègne, en réaction à la décision du tribunal de Sarreguemines déboutant les représentants du personnel de leur demande d'annulation du plan social et à l'impossibilité de tenir la réunion tripartite demandée. Le «saccage» des bureaux est filmé en direct et diffusé sur les ondes dans les heures qui suivent. Peu après, la destruction du local de garde placé à l'entrée de l'usine et la détérioration du système de sécurité constituent un point de non-retour dans le conflit puisque ces faits sanctionnent l'impossibilité matérielle de retourner à son poste de travail.

Ces actes signent bien une seconde phase de la mobilisation. Ils marquent un engagement non seulement plus politique mais aussi ostensiblement plus contestataire dans la démonstration d'usages symboliques de la violence. Du statut médiatique de «victime» à celui de «délinquant», le pas est très vite franchi. Les actes de dégradation d'éléments de mobilier de la sous-préfecture sont, en effet, aussitôt dénoncés par le gouvernement Fillon qui promet de poursuivre les responsables ${ }^{(19)}$. Ces derniers sont bientôt vilipendés par une partie de la gauche et certains responsables syndicaux qui voient dans la présence de Roland Szpirko sur le site de Clairoix la main séditieuse de l'extrême gauche(20). Cette seconde phase du mouvement est aussi symbolisée par l'émergence de Xavier Mathieu, ouvrier cégétiste, promu porte-parole du collectif. La fascination de la presse pour la personnalité de celui qu'elle présente comme le leader de la lutte contribue également, sans nul doute, à la populariser et à en faire le récit. La presse construit, à l'image du

(18) «La droite renvoyée à ses promesses», Le Courrier picard, 17 mars 2009.

(19) «Fillon promet des poursuites», Libération, 23 avril 2009. (20) «L'ami d'Arlette chez les Continental», Le Journal du dimanche, 3 mai 2009. portrait publié dans Libération le 31 août 2009, une figure modernisée du héros ouvrier, à la fois romantique et révolté. Or, réduire ce mouvement social à un binôme de leaders charismatiques - certes, Xavier Mathieu et Roland Szpirko sont les plus exposés médiatiquement - ne rend pas compte des logiques et dynamiques collectives qui l'ont rendu possible. D'autres militants mais aussi des salariés non-syndiqués ont, souvent dans l'ombre, œuvré au plus près à l'encadrement et à l'organisation des actions. Certains, comme Jacques Pichon et Patrick Lachambre de la CGT ou François Lefèvre de Force ouvrière sont très actifs et assurent l'intendance du mouvement, tandis que d'autres travaillent à la mobilisation de leurs compagnons de lutte.

\section{Une lutte dans la lutte: imposer "un syndicalisme de combat"}

Dès les premiers instants suivant l'annonce de la fermeture, des tensions entre les syndicats sont visibles. Comme le dit Julien Robin en janvier 2014, le responsable de la CFTC va très vite se trouver débordé par la situation :

«La CFTC qui était majoritaire, a été complètement discréditée, complètement discréditée. [...] S'il avait dit : "J'ai signé l'accord mais..." Mais à chaque fois il faisait un tract, il tenait avec la direction. Alors au moment où on annonce la fermeture, il n'avait plus d'arme. Au début du conflit, c'était très chaud pour lui, c'était le traître! Il a été pris comme un traître.»

Appelant à la discussion, le délégué CFTC ne parvient pas à contenir le mouvement de la foule rassemblée dans l'usine, lorsque le directeur du site vient confirmer la nouvelle à l'équipe de l'aprèsmidi. Il y voit les tactiques habiles de la CGT pour l'écarter en le rendant complice de la duperie patronale pour avoir signé les «quarante heures» :

«Le fait de mettre "pour la pérennité du site et des emplois" dans l'accord des quarante heures en 2007, c'est là où j'ai fait une erreur, parce que dans la tête des gens, c'était: "On a fait un accord, l'usine ne peut plus fermer" ... et c'est là que la CGT arrive très fort et dit : "Je vous l'avais dit, il était dans le coup... il savait que l'usine allait fermer, c'est pour ça qu'il vous a fait passer à quarante heures" ... Trop fort! Ça, dans la nuit noire, dans la foule, avec les spots, vous êtes mort, vous êtes mort. D'un seul coup, je suis presque le complice de la direction...»

(Entretien avec José Munes, délégué CFTC, réalisé en janvier 2014.) 
De fait, il perd irrémédiablement tout crédit auprès de la plupart des Conti. Michel Bonte, délégué FO, dénonce en octobre 2013 : "C'était une trahison complète." François Lefèvre, technicien qualité, autre délégué $\mathrm{FO}$, explique : «Le fait d'être repassé aux quarante heures, les gens l'avaient en travers de la gorge. On te dit que t'es tranquille jusqu'en 2012 et un an après on ferme! " Quant au responsable de la CFDT, il a été le premier à alerter sur le risque de fermeture, mais il savait n'avoir aucune chance objective de prendre le «leadership» étant donné sa position hiérarchique : "Moi je faisais partie de l'encadrement. Dans une boîte comme cela, il y a 1000 ouvriers et ce n'est pas un cadre qui peut prendre la parole. " La CGT, pour sa part, adopte une position radicale, appelant l'ensemble du personnel à se mobiliser. Suivie par Force ouvrière, elle organise une assemblée générale dès le matin de l'annonce de la fermeture de l'usine et tente de préparer la riposte. Toutefois, elle ne dispose que d'une faible base militante, pas très organisée et, surtout, ne possède pas les ressources pour construire un rapport de force consistant et durable face à la direction qui a pris les salariés de cours. Le besoin des conseils d'un stratège, expérimenté dans ce genre de conflits, explique, sur la forme, l'arrivée de Roland Szpirko (voir encadré 4).

La recherche, à ce point de l'enquête, ne peut établir de faits convergents sur les discussions qui ont présidé à sa venue. La célérité avec laquelle il intervient, dès les premières heures, laisse supposer que des relations préexistaient au mouvement. Elles étaient assez solides pour être mobilisées dans l'urgence, certainement par l'entremise de Jean-Marc Iskin, qui avait gardé des contacts avec la CGT de l'usine et qui a été régulièrement présent sur le site durant le mouvement. Il n'est pas établi, par contre, que les représentants élus CGT fréquentaient les réseaux de Roland Szpirko. Xavier Mathieu est souvent décrit comme protestataire et libertaire, avec «la fibre écolo». D'autres, comme Patrick Lachambre notamment, sont proches du Parti communiste qui compte nombre d'élus dans le Compiégnois (conseiller régional ancien député, conseillers généraux, maires). La présence de Roland Szpirko est d'ailleurs de nature à tendre ces relations de proximité construites depuis longtemps. Néanmoins, en raison de son expérience des luttes, il apparaît comme un allié de poids, doté d'un savoir-faire militant mobilisable face à une situation exceptionnelle devant laquelle les syndicalistes se sentent démunis. Xavier Mathieu le reconnaît quelques mois plus tard dans la presse : «Tout seul je ne me sentais pas capable de faire face au conflit ${ }^{(21)}$.» Michel Bonte, un des représentants de FO, approuve cette initiative même si, au début, il est un peu réticent :

"J'étais une quiche comme les autres... et voilà le conflit, je n'avais pas les épaules. Je vais te dire

(21) «Un homme en colère», Libération, 31 août 2009. franchement, moi, une stratégie, j'en avais aucune. Je représentais la FO mais je n'avais aucune stratégie. Tu prends tout ça en pleine gueule... c'est pour cela quand Roland est arrivé, c'est lui qui a donné la marche à suivre. "

(Entretien réalisé en octobre 2013.)

\section{Encadré 4}

\section{L’arrivée de Roland Szpirko : un militant Lutte ouvrière chez les Conti}

Engagé en politique depuis sa jeunesse, Roland Szpirko est devenu une figure politique du département de l'Oise, en particulier dans l'agglomération creilloise. II est élu au conseil municipal de Creil depuis 2008 et siège un temps au conseil régional de Picardie. Roland Szpirko arrive dans l'Oise en 1978 , au moment où il est embauché comme ouvrier à l'usine Chausson (1). Militant révolutionnaire, il s'engage rapidement sur le plan syndical, à distance des confédérations ${ }^{(2)}$. Mais c'est surtout durant le conflit social qui se développe au moment de la fermeture de l'usine que cet ouvrier militant se fait connaître du grand public (3). Renouant avec des méthodes «d'action directe» (PIGENET, 2012), les salariés de Chausson investissent la Bourse et le plateau de France 2 (1993), occupent le siège national de l'ANPE à Noisy-le-Grand ou la direction des impôts de Creil (1995). Au terme de trois années de combat, ils parviennent à arracher un plan social que d'aucuns qualifient d'exemplaire (LinHART et al., 2002; MAssera, Grason, 2004).

Peu médiatisée au niveau national, cette lutte a néanmoins constitué une référence pour nombre de syndicalistes et notamment pour ceux de l'usine Continental. Xavier Mathieu y fait d'ailleurs référence dans un propos rapporté par le journal Libération : «Si les Chausson ont obtenu ce qu'ils ont obtenu, c'est grâce à lui (4). " Les journalistes commencent, en effet, à s'intéresser à la présence de cette figure emblématique de la lutte Chausson et tenant d'un syndicalisme radical. Le problème est souvent posé en termes d'infiltration ou d'instrumentalisation des mouvements sociaux par des organisations d'extrême gauche. Or, la question n'est pas tant, ici, de savoir si Lutte ouvrière a «noyauté» les Conti que de se demander ce qui lui a permis d'être au cœur de cette lutte. II s'agit alors de s'interroger sur les raisons de son arrivée pour tenter de comprendre comment les salariés l'ont suivi.

(1) L'entreprise Chausson de Creil était une usine de production automobile appartenant au groupe éponyme, filiale de Renault et Peugeot. Il fut le plus grand site industriel de Picardie avec, dans les années 1970, près de 5000 salariés.

(2) Après avoir été exclu de la CGT puis de la CFDT, il crée avec un carré de fidèles le Syndicat démocratique Chausson (SDC) dont l'audience culmine à $21 \%$ des suffrages aux élections professionnelles de 1990.

(3) Ce conflit touche l'ensemble du groupe Chausson mais l'essentiel des actions est mené par les salariés du site de Creil, condamné à fermer. Si quelques responsables syndicaux de Gennevilliers, comme Bernard Massera, représentant au CCE, appuient cette lutte, Roland Szpirko en est, localement, l'un des principaux animateurs.

(4) «Trois combats emblématiques», Libération.fr, 5 mai 2009. 
Au-delà des étiquettes syndicales, l'engagement dans le conflit repose sur des hommes qui ont déjà appris à se connaître et construit des solidarités dans le travail. Quant aux représentants élus de la CFTC et de la CFDT que nous avons interviewés sur ce point, la présence de Roland Szpirko fut, pour eux, une surprise. Mis devant le fait accompli, réprouvant à des degrés divers cette initiative (Dif-Pradalier, Reix, 2012), ils apparaissent dès lors en retrait tandis que Force ouvrière est beaucoup plus impliquée.

L'intégration de Roland Szpirko au Comité de lutte, votée à la quasi-unanimité en assemblée générale, lui ouvre la possibilité de participer aux instances de direction qu'il va contribuer à élaborer. Il apporte un répertoire d'actions et une lecture politique de la situation. Les manifestations constituent, chaque fois, à la manière de celles des Chausson dans les années 1990, de véritables démonstrations de force. Cela est le cas, par exemple, à Sarreguemines, le 6 mai 2009, pour obtenir une date de réunion avec la direction de Continental et l'État ou, un peu avant, lors du voyage à Hanovre, le 23 avril, à l'occasion de l'assemblée générale des actionnaires :

«L'Allemagne... c'était un grand moment! Est-ce que tu crois qu'on aurait pensé à ça ... qu'est-ce qui a pensé à ça? C'est le petit grain de sable! Ne serait-ce que l'idée de le faire. Nous le combat, il se menait ici. Quand il nous dit, il faut un train pour l'Allemagne, je me dis, qu'est-ce qu'il nous fait encore?"

(Entretien avec Michel Bonte, représentant FO, réalisé en octobre 2013.)

Le succès et la médiatisation des premières mobilisations galvanisent les Conti, renforçant l'idée que la stratégie de Roland Szpirko est payante. " Si on n'avait pas eu cet homme-là, je pense qu'on n'aurait pas eu ce qu'on a obtenu», dit aujourd'hui Robert Jaquin.

\section{Une critique par le "bas" des confédérations}

On peut observer de fortes similitudes entre la lutte des salariés de Chausson du début des années 1990 et celle des Conti. La particularité du mouvement des ouvriers de Creil tenait tout autant à la radicalité des formes d'actions qu'aux façons de les organiser. Dans l'effervescence liée à la réussite des opérations de début 1993, les militants du SDC (Syndicat démocratique Chausson) étaient parvenus à imposer aux autres organisations syndicales leur conception de la grève. Ils avaient pu mettre en place des méthodes de conduite du mouvement rompant avec la représentativité établie, le dotant d'un véritable organe exécutif qui poursuivait un double objectif : une appropriation de la lutte par les acteurs et, tout autant, son autonomisation par rapport aux organisations établies, soumises à l'influence des instances fédérales. Le Comité de lutte des Conti qui dirige le mouvement social de 2009 est construit sur le modèle retenu à Creil en 1993 :

"C'est la stratégie de Roland. C'est tenir des assemblées générales régulières, tenir au courant tout le monde. Ce n'est pas les syndicats, les fédérations qui décident pour les ouvriers. C'est-à-dire que tout le monde était acteur du truc, chaque ouvrier était acteur de son destin. [...] Ce n'est pas moi, Jacques ou Xavier qui décidait pour l'ensemble du personnel, c'était tout le monde qui décidait pour tout le monde. $C$ 'est pour ça qu'on avait 800 personnes. Tout ça, c'est bien simple, c'est Roland qui l'a amené. »

(Entretien avec Michel Bonte, représentant FO, réalisé en octobre 2013.)

Aussi, quand la discussion s'est engagée sur l'opportunité de recourir aux services de Roland Szpirko, il s'agissait en réalité de débattre, pour reprendre les mots de Pierre Bourdieu (1984), de la légitimité des moyens à employer. Devant l'enjeu de la situation, la section CGT de Clairoix parvient assez facilement à imposer le modèle d'action collective proposé par Roland Szpirko : "Il avait déjà géré un plan social chez Chausson, il a expliqué plein de choses et on est arrivé à mettre en place plein de choses », justifie Robert Jaquin.

Durant les quatre mois que dure le conflit, un groupe composé de plus d'une cinquantaine de volontaires impliqués dans le comité se réunit dans les locaux du réfectoire pour discuter la stratégie de lutte et décider les actions qui sont ensuite proposées aux assemblées générales. Contestant la représentativité établie, le Comité de lutte entend avoir la maîtrise de l'organisation du mouvement et des négociations avec l'État. La présence d'hommes réfractaires aux ordres et aux instructions dictés «d'en haut», comme Xavier Mathieu, contribue à autonomiser les décisions du groupe des injonctions confédérales. La création de ce comité, dès le début du conflit, et le poids qu'il va bientôt prendre font éclater l'intersyndicale qui s'était constituée dès les premiers moments du conflit. Elle permet également au militant de Lutte ouvrière d'entrer dans le jeu et de porter ce mouvement dans le champ politique. Si la CFDT et la CFTC refusent de reconnaître la légitimité de cette organisation, le représentant de la CFE-CGC lui apporte son soutien et, plus globalement, celui des cadres.

L'expérience des premières semaines conduit les Conti à prendre la mesure de la portée politique de leur mouvement. Les plus engagés vont beaucoup apprendre de leur confrontation avec ces univers du pouvoir, jusque-là totalement méconnus, et découvrir la réalité des règles de la négociation. Non seulement leur rencontre avec le conseiller social de la présidence de la République, le 25 mars 2009, ne permet aucune avancée sur l'organisation de la réunion tripartite qu'ils demandent, mais ils ont en outre l'impression que leurs interlocuteurs font preuve d'un comportement empreint de duplicité. 
Michel Bonte, délégué FO nous 1'explique en octobre 2013 : "Ils nous disent qu'ils n'ont pas de contact avec Continental et pendant ce tempslà Continental était en réunion avec Chatel... donc voilà... et on l'a appris par des journalistes. » De même, les manœuvres des centrales syndicales au moment de la préparation de la rencontre conduisent à un incident sérieux avec une base déjà soupçonneuse. Michel Bonte raconte l'épisode :

«On réussit à décrocher une réunion avec Raymond Soubie. Donc, D [un des responsables de la section FO] a un coup de téléphone et il me dit: "Michel, toi tu ne viens pas, $H$. le fédé chimie, le boss de la fédé chimie FO nationale, il me l'a dit." Je téléphone, je lui dis :

- C'est quoi tu veux prendre ma place!

- Oui, je suis secrétaire de la fédé chimie, ça a été décidé.

- Tu viendras me le dire devant les 900 Conti que tu prends ma place, à moi délégué, alors que vous ne nous avez jamais apporté le moindre soutien depuis le début du conflit.

A côté, Xavier [il évoque ici l'appel reçu par Xavier Mathieu au même moment], c'est le patron de la fédé chimie de la CGT. Et Xavier a démarré au quart... "On ne vous a jamais vus." Dring CFDT, rebelote.... CFE-CGC .... Le même jour à cinq minutes d'intervalle, c'était impressionnant! Tous ces gens-là, on aurait dit qu'ils étaient en réunion avec je ne sais qui et tous dans le même bureau. Ils téléphonaient un à un et ça se trouve avec le même téléphone! [...] Nous on s'est dit, il faut leur couper l'herbe sous le pied. Et c'est ce qu'on a fait, mais crois-moi, encore aujourd'hui, c'est difficile de communiquer avec la fédé. »

Les responsables fédéraux, informés avant les Conti de la réunion à l'Élysée, tentent de s'imposer à la table de négociation en déléguant un représentant de chaque confédération. La volonté des centrales syndicales est, selon les sources de Julien Robin (responsable CFDT), de prendre la main sur un mouvement social qui leur échappe, de contrôler Xavier Mathieu et de neutraliser l'action politique de Lutte ouvrière. Devant l'hostilité du Comité de lutte, personne ne prévoit de participer à la réunion de l'Élysée, à l'exception d'un responsable fédéral de la CFDT, mais qui préfère finalement renoncer. Cet épisode est un moment crucial du conflit. Pour les membres de la CGT et de FO, il vient non seulement justifier les mises en garde du militant de Lutte ouvrière mais lui donne également totalement raison sur la stratégie à poursuivre. L'échec du projet des fédérations est vécu par les responsables locaux de la CFDT et de la CFTC comme le signal d'une impossibilité de reprendre la main sur la direction du mouvement. Dès lors, l'action syndicale procède d'un renversement de pouvoir : la CGT et FO, minoritaires, tiennent, à travers le Comité de lutte et son porte-parole charismatique, les rênes de la contestation, tandis que la CFTC, syndicat majoritaire, reste l'allié objectif incontournable de tout accord négocié et signé.

Si la présence de Roland Szpirko a conduit à tendre les rapports avec les confédérations syndicales, l'incident relaté ci-dessus officialise la rupture. Les liens avec les grands leaders nationaux de la CGT et de FO deviennent alors des plus distants, voire inexistants. Seul le secrétaire général de la CFDT, François Chérèque, vient dans l'Oise soutenir ses cadres locaux un peu ébranlés par la tournure des événements, mais ne se déplace pas sur le site. La rupture, consommée dès les premiers moments du conflit, apparaît au grand jour quelques mois plus tard en août 2009, quand Xavier Mathieu, interviewé sur les ondes de France info, utilise le terme de «racaille» pour dénoncer le manque de soutien des instances syndicales à la cause des ouvriers de Clairoix. Au-delà des mots qui témoignent un fort ressentiment, le propos révèle surtout que la base militante se sent en déphasage complet avec des organisations bureaucratiques qu'elle perçoit éloignées des terrains de lutte et dont elle conteste l'approche. La lutte des Conti et la mobilisation plus large qui se construit autour d'elle montrent que les luttes dites «défensives » ne se traduisent pas nécessairement par un repli sur soi. Les manifestations et meetings organisés durant les trois mois de conflit sont le théâtre de rencontres avec d'autres salariés en lutte, aux appartenances syndicales diverses et souvent en désaccord avec leurs centrales. Ces temps forts engagent également des militants associatifs et politiques qui dénoncent à la fois le capitalisme et la pénalisation de l'action syndicale, à la suite des poursuites engagées contre les sept salariés de Continental pour les dégradations commises à la sous-préfecture de Compiègne.

Soucieux de leur indépendance, les Conti prennent soin, en dépit du désaccord de leur allié de Lutte ouvrière, d'inviter aux événements qu'ils organisent les dirigeants locaux et nationaux des partis politiques de gauche, toutes tendances confondues. Ils souhaitent neutraliser les effets d'un mouvement trop marqué à l'extrême gauche, en offrant l'image d'un vaste rassemblement d'opposition à la majorité présidentielle. L'expérience de ce conflit est aussi un temps de socialisation politique qui débouche sur une critique sociale forte, portant à la fois sur les nouvelles formes de la domination capitaliste et sur les structures militantes traditionnelles qui leur apparaissent inopérantes. Un an après la fermeture, en octobre 2010, alors que les salariés sont encore en congé de mobilité, de nouvelles élections professionnelles sont organisées afin de renouveler les représentants du personnel. Même si l'usine est fermée, le contrat de travail n'est pas encore rompu. À l'exception des salariés ayant refusé d'entrer dans le dispositif de reclassement (environ 
70 personnes), la base électorale est inchangée (22). Les candidats de la CGT et de FO qui ont fait liste commune obtiennent près de $66 \%$ des voix aux élections au comité d'entreprise. Les syndicats qui ont été en retrait durant ce mouvement (CFTC et CFDT) perdent la plupart de leurs représentants. La CFTC, majoritaire depuis 2004, recueille 27\% des voix et voit disparaître près de la moitié de son électorat, alors que la CFDT ne parvient pas à franchir la barre des $10 \%(7 \%)$. Les résultats de ce vote donnent une écrasante majorité aux animateurs du Comité de lutte, semblant valider à la fois la stratégie et les actions retenues durant le conflit. Comment le discours politique a-t-il rencontré les attentes de la majorité des salariés ?

\section{Les ressorts de la mobilisation}

Comme d'autres recherches sur la conflictualité sociale ont pu le montrer, lors de la fermeture des usines Chausson (Linhart, 2005), Cellatex (LARose et al., 2001) ou encore Moulinex (RouPNEL-FuENTES, 2011), l'effet de sidération produit par l'annonce du projet de fermeture s'accompagne d'un sentiment de duperie. Les salariés de Continental se sentent doublement trompés par une direction qui leur a promis de maintenir la production en contrepartie de l'accord de 2007 au titre duquel, pendant deux ans, ils ont consenti des efforts supplémentaires. Ils ne se font guère d'illusion sur l'avenir de leur site même si, secrètement, ils gardent en eux un espoir, tellement cette décision leur paraît inconcevable. L'usine est en effet un outil de production moderne, doté des machines les plus sophistiquées : "C'était robotisé... c'est des trucs qui valaient des... Quinze jours avant on montait encore des robots dans l'usine! Je n'ai pas compris... la veille un gars se faisait embaucher dans l'usine et du jour au lendemain on nous dit on ferme!", s'indigne encore aujourd'hui Robert Jaquin, l'ancien ouvrier de production. Il nous parle ensuite de la difficulté à faire face à la fermeture et à la perte de son emploi : "Heureusement que j'ai une femme qui m'a soutenu! Heureusement, parce que c'est dur à vivre... J'ai pensé plus d'une fois à sauter d'un pont... perdre son emploi, surtout Continental... Passer trente ans de sa vie, c'est beaucoup hein!» Les messages qui accompagnent le retour des questionnaires réalisés dans le cadre de l'enquête sur le reclassement disent la même chose, un ressentiment encore très présent, qui se fige désormais, pour bien des Conti, dans l'instabilité de la précarité et du chômage. La blessure reste profonde et térébrante, comme "une grande cicatrice ouverte pendant longtemps », note dans le questionnaire

(22) Le comité d'établissement poursuit son activité jusqu'en février 2014, date du licenciement des salariés protégés. renvoyé en janvier 2013 Éric Tallant, technicien de maintenance, 45 ans.

\section{Un monde qui s'écroule, une injustice inacceptable}

Leur monde - constitué en grande partie par l'histoire commune et collective du travail et les valeurs qui y sont attachées - s'écroule comme un château de cartes, à l'image de ce qui s'est passé ailleurs (Linhart et al., 2002; Burgi-GoluB, 2002; Magloire, 2002; Fajardie, 2003; Goergen et al., 2007). Dans notre recherche, le désarroi et l'anéantissement sont évoqués dans de nombreux témoignages recueillis en marge des manifestations et assemblées générales. Ils se lisent aussi dans un espace que nous avons laissé ouvert aux commentaires dans l'enquête par questionnaires de janvier 2013 : "Le mental en a pris un coup», concède Alain Marchand, ouvrier polyvalent de 49 ans. "Depuis la fermeture de l'usine, ma vie s'est effondrée, j'ai connu le divorce, l'invalidité», confie Jean-Pierre Leullier, opérateur tringles de 42 ans, ou encore, «Je suis rentré chez Continental par la grande porte et je suis sorti par la petite porte!», ironise Marc Doré, magasinier de 56 ans, trentedeux ans d'ancienneté. Quel bilan tirer de cette fermeture? "Pour moi c'est une grande perte, un gâchis! Retrouver un emploi comme Continental! $C$ 'était dur les $3 / 8$, les $4 / 6$ mais on avait des primes de vacances, intéressement, mutuelle. Maintenant pour retrouver cela, on ne retrouvera plus jamais », résume Robert Jaquin. Néanmoins l'unité du mouvement ne s'exprime pas par le champ lexical de la souffrance. Elle est plutôt nourrie par le sentiment d'injustice et l'idée insupportable d'avoir été manipulé, humilié et déconsidéré socialement. C'est, pour les Conti, le «contrat moral» pour lequel ils se sont engagés subjectivement dans le travail qui n'a pas été respecté :

«Depuis le licenciement, le moral a souffert. Les recherches sont quasiment néantes et mon âge ne m'aide pas! [...] L'avenir est incertain... Les patrons veulent tout et surtout ne plus payer les efforts et les sacrifices que fait un ouvrier. »

(Philippe Perrin, ouvrier confectionneur, 56 ans, six ans d'ancienneté, travail posté en week-end. Enquête par questionnaires de janvier 2013.)

«J'ai perdu $1200 €$ nets par mois. [...] L'Europe $n$ 'aurait jamais dî exister! La preuve: Continental est parti en Roumanie sans aucun scrupule pour les personnels qui leur ont fait gagner des bénéfices énormes!»

(Stéphane Pillon, cariste puis agent polyvalent, 45 ans, sept ans d'ancienneté, travail posté en week-end. Enquête par questionnaires de janvier 2013.)

Les discours dénoncent aussi bien la brutalité de la fermeture qui les met en danger socialement et économiquement que le mépris d'une direction qui 
ne reconnaît pas le mérite attaché au travail et ne règle pas correctement ce qu'elle doit.

«C'était ma deuxième maison, j'étais bien là-bas. Il y avait des horaires, des inconvénients mais moi, Continental, moi je pensais qu'une boîte comme cela, je pensais terminer jusqu'à ma retraite là-bas. »

(Entretien avec Robert Jaquin en janvier 2014.)

Au tournant des années 1990, le passage à «l'entreprise néolibérale» (CоUтRot, 1998) rend caduc le «compromis fordiste» sur lequel était fondée la société salariale et qui avait réussi à pacifier les relations de travail après la seconde guerre mondiale. Le paysage industriel est alors transformé par un mouvement de restructurations et de rachats incessants, qui imposent un nouvel ordre productif. Le management rappelle ainsi aux salariés que leurs unités de production sont susceptibles, du jour au lendemain, de changer de propriétaire ou de fermer purement et simplement si la rentabilité n'est pas conforme aux attentes des conseils d'administration (FAVIER, 2008). Qu'est-ce qui est en jeu dans ces fermetures?

Comme l'a rappelé Axel Honneth (2000), le travail est constitutif d'un système de reconnaissance qui permet aux individus, à partir de la contribution qu'ils apportent, de se sentir appartenir à une société. L'acte professionnel se trouve ainsi au cœur d'un processus par lequel les travailleurs attendent en retour non seulement un salaire, établi contractuellement, mais également des marques d'estime sociale (RENAULT, 2004). Le consentement au travail est aussi fondé, comme le montrent les témoignages précédents, sur des usages, des conventions, des obligations réciproques, des codes implicites, bref sur toute une «économie morale» pour reprendre le fameux concept de THOMPSON (1988a). Les analyses de l'historien britannique peuvent nous aider à poursuivre la réflexion. Étudiant les révoltes populaires du XVIII et début du XIX ${ }^{\mathrm{e}}$ siècles en Angleterre au moment où se constitue le capitalisme industriel, il propose des hypothèses qui contestent les approches d'un marxisme vulgaire. Pour lui, les conditions matérielles ne peuvent pas à elles seules expliquer les raisons pour lesquelles les individus se révoltent contre ceux qui les dominent. Il s'intéresse ainsi aux représentations, aux émotions, aux façons dont sont appréhendées subjectivement les situations. Contre le "réductionnisme économique», il suggère une lecture plus anthropologique pour tenter de saisir de l'intérieur les mobiles réels de ces luttes (1988a, p. 31). Pour le dire autrement, les révoltes peuvent naître non seulement de la raréfaction des ressources ou d'une baisse des moyens matériels d'existence, mais aussi du sentiment que les règles, normes ou engagements n'ont pas été respectés (FASSIN, 2009).

À la suite de l'annonce faite par la direction dans l'entrepôt, les réactions ont été, en premier lieu, d'ordre émotionnel, se rappelle Robert Jaquin. Certains ouvriers, ne parvenant pas à masquer leur douleur, s'effondrent; d'autres s'enfuient pour se mettre à l'abri des regards. L'expérience sociale du mépris va rapidement se fondre en colère. Robert Jaquin explique en janvier 2014 comment il a réagi à l'époque :

"Au début, c'est de la colère parce que l'usine ferme et que je savais qu'elle faisait des bénéfices. Si elle était en déficit et en dépôt de bilan, OK, on aurait compris. Mais là on ne peut pas comprendre qu'une boîte comme Continental, une grosse boîte comme ça elle ferme. Quand on fait des bénéfices, on ne doit pas foutre en l'air des gens comme cela!»

L'injustice se situe, ici, à deux niveaux. Le premier est celui de l'arbitraire. Pour l'ouvrier, la rentabilité avérée de l'entreprise ne peut, en aucun cas, justifier la fermeture. Continental est un groupe industriel prospère qui engrange chaque année des profits conséquents. Corrélativement, cette fermeture lui apparaît d'autant plus illégitime que la rentabilité du site est le fruit de l'engagement productif des salariés, au risque de leur santé et de leur intégrité physique. Le second niveau est celui de la déloyauté à l'égard des salariés. Non seulement le groupe industriel ne se soucie pas du devenir de ceux qui, à son service depuis des années, vont perdre leur statut social, mais de plus, il leur inflige une humiliation publique par l'annonce brutale, sans préavis, de leur licenciement. Ces deux niveaux d'injustice vont prendre corps dans le sentiment de trahison envers les engagements contractés avec «l'accord des quarante heures » : le «contrat moral» est rompu.

\section{Du registre de la dignité à la lutte des classes}

Peu après l'annonce faite dans l'usine, les salariés se retrouvent à l'extérieur et chacun tente d'avoir des informations complémentaires. Ils sont abasourdis par la nouvelle; le spectre de la fermeture si souvent agité est devenu réalité. Robert Jaquin raconte: «On se retrouve dehors, les gens disaient : putain tu te rends compte!» La résistance, qui commence à s'organiser, trouve sa légitimité dans la conviction, bientôt partagée par toutes les catégories de salariés, de devoir se dresser ensemble contre la direction. François Lefèvre, syndicaliste FO, précise en octobre 2013: "Ils ont annoncé la fermeture d'usine totale. Tout le monde dans la même merde, les ouvriers, les Etam, les cadres. " Lors des assemblées générales, Xavier Mathieu a souvent recours à la sémantique de la dignité, appelant à la justice sociale et dénonçant le mépris de classe. En mobilisant ainsi les répertoires de l'expérience collective, l'enjeu est, ici, de construire un rapport de force pour «ne pas, dit-il, crever comme des chiens ». Le travail militant consiste à faire en sorte que la conscience d'injustice, la «conscience de tort» (HoNNETH, 2000) prenne une dimension sociale et politique. Robert Jaquin la manifeste en ces termes : «C'est comme cela la boîte, elle est fermée et on ne reviendra pas en arrière, c'est comme cela, c'est là-haut qui a décidé! Que voulez faire à part être mécontent, casser, 
balancer des œeufs, casser... la colère c'est ça, il faut l'exprimer!» Mobilisant les émotions sur le registre de l'indignation, de la révolte, les syndicalistes de la CGT leur donnent un sens collectif, et, en corollaire, se sentent pleinement légitimes à conduire le mouvement social. C'est grâce à leur capacité à canaliser la colère et à l'inscrire dans un schéma de compréhension et d'action que les salariés ont pu se rassembler et agir dans le même sens ainsi que l'indique Robert Jaquin : "Après, il a bien fallu qu'on se batte. Et heureusement qu'on s'est battu, sinon on n'aurait pas eu tout cela, le congé de mobilité, les primes, les mesures d'âge, l'accompagnement.» Le premier combat des syndicalistes est donc de faire reconnaître auprès de leurs compagnons de travail le bien-fondé de la lutte et de lui donner un objectif.

Parallèlement, comme le font observer les membres du Comité de lutte lors des entretiens que nous avons réalisés avec eux, il leur a fallu rapidement être en mesure de socialiser la colère et d'éviter tout débordement: "La colère des gens était légitime mais nous, on a essayé de temporiser", précise Michel Bonte avant de poursuivre, "s 'il y a un truc qu'on a fait, c'est d'éviter la casse parce que, regarde l'usine, ç'aurait été un tas de cendres». Rappelant la grande tension qui régnait à l'époque, François Lefèvre dit la même chose: "C'est nous qui avons empêché les gens de tout broyer. Mais c'est vrai que si les machines sont parties aussi belles qu'elles sont arrivées... si on n'avait pas été là, ça explosait.» Dans ces moments de crise, le rôle des délégués, précisent nos interlocuteurs, est de travailler à la mobilisation tout en œuvrant à contrôler au mieux la situation afin d'éviter tout incident. Comme le montre LAROSE (2001) à propos de Cellatex, le syndicat a un rôle de pacification d'autant plus, note-t-il, que «les salariés n'ont confiance qu'en leurs luttes et ne croient qu'aux rapports de force» (p. 16). Aussi, l'enjeu des premiers jours a été de construire un cadre suffisamment structurant, à la fois pour maintenir une cohésion collective et se constituer en partenaires de négociation crédibles aux yeux de la direction, qui pouvait craindre la dégradation de son parc machines, et d'un État soucieux de l'ordre public. Les processus de légitimation de l'action collective ainsi que le sort qui lui est réservé dépendent étroitement de la manière dont elle se manifeste dans l'espace public et de l'environnement social et politique. Autrement dit, il faut également que l'injustice soit reconnue socialement injuste. À cet égard, le contexte de 2009 s'est caractérisé par un soutien populaire aux luttes sociales, même les plus radicales, comme le montre notamment une enquête de l'Ifop ${ }^{(23)}$.

(23) «Les Français et la séquestration des patrons», sondage réalisé par l'Ifop (Institut français d'opinion publique) en avril 2009, commandité par le journal L'Humanité. Ce sondage note que $63 \%$ des personnes interrogées disent comprendre les actions de séquestration des patrons; la moitié d'entre elles les approuvent même.
Mais cette légitimité sociale reste fragile. Elle est soumise au moindre incident susceptible de dégénérer ou au moindre jugement dépréciatif sur le bien-fondé de la lutte, d'autant plus que celleci constitue un enjeu politique de premier plan. Ainsi, les Conti ont-ils pu être perçus, parfois même de la part de militants, plus enclins à négocier des indemnités de licenciement substantielles qu'à lutter pour le maintien de l'emploi, contrairement à ce qui s'est passé lors d'autres conflits, comme celui de Goodyear à Amiens notamment (Dif-Pradalier, Reix, 2012). Cette critique, qui s'appuie sur le registre moral (les ouvriers doivent se battre pour le travail, non pour l'argent), a pu, en corollaire, comme lors des événements survenus à la sous-préfecture, concourir à délégitimer le mouvement. L'obtention de la prime extralégale de $50000 €$ et du congé de mobilité de deux ans a certainement aussi contribué à brouiller le message en présentant les Conti comme des privilégiés. "Nous, ce qu'on voulait, c'est que l'usine reste ouverte », se défend Michel Bonte en octobre 2013 avant de poursuivre : "Le combat, ce n'était pas les primes mais que l'usine reste ouverte et vive. Mais par contre, on a été vite calmé avec la décision du tribunal de Sarreguemines... Là, le rouleau compresseur était en route... Donc ce jugement, ça a été notre mort.» "La cause de l'emploi» (Didrr, Teyssier, 1996) s'est, ici, construite sur le registre moral de la «trahison », du non-respect des engagements pris par la direction du groupe Continental. Si l'argument a permis de constituer un rapport de force et d'inscrire le mouvement dans la vague de contestations de 2009, aucun élément tangible, aux yeux de la justice, ne permettait de justifier l'annulation de la procédure de licenciement collectif. La décision du tribunal de Sarreguemines, le 22 avril 2009, rejetant le recours engagé par le comité d'établissement, a non seulement ruiné les espoirs d'arrêter le plan social, mais a aussi lancé le compte à rebours. Sans recours possible devant les juridictions pour arrêter le processus de fermeture, les Conti se sont alors battus pour obtenir des garanties matérielles (indemnités et congé de mobilité) suffisamment étendues afin de mettre à l'abri de la précarité, le plus longtemps possible, les salariés les plus fragiles. Mais au-delà, une fois la fermeture entérinée, il s'agissait de faire payer le plus cher possible le «mépris social» en évaluant le niveau de réparation du «préjudice moral» et des dommages sociaux subis.

L'idée d'une dette incommensurable, jamais soldée, pour reprendre l'image développée par Sylvie MALSAN (2007) au sujet des ex-salariées d'Alcatel, attise le ressentiment et le besoin de justice sociale. Pour les Conti, le mouvement social qui se construit dès mars 2009 confère progressivement à ses représentants une double responsabilité : promouvoir à la fois les attentes de réparation (matérielle) et la 
demande de considération (symbolique) du groupe humilié. L'expression collective qui se déploie alors dans la sphère publique s'adosse à un éventail de revendications matérielles tout en dénonçant l'exploitation capitaliste qui se caractérise par un déni de reconnaissance et l'inégale redistribution des bénéfices (Fraser, 2005). Les propos tenus dans le questionnaire, bien après la fin du «temps chaud» du mouvement, portent encore les traces de la radicalisation politique :

«C'est une France de merde, des capitalistes à brûler [... Je suis] un citoyen usé par le travail... qui se voit aujourd'hui comme un Kleenex. Vive les Conti!!!»

(Marc Maupin, opérateur de fabrication, 56 ans, dix ans d'ancienneté, travail posté en $3 / 8$.)

"Pour l'enrichissement de quelques-uns, on n'hésite pas à sacrifier des centaines de familles!!!»

(Lionel Toupart, dépanneur en 4/6 puis polyvalent posté en $3 / 8,50$ ans, quinze ans d'ancienneté.)

"Continental n'avait aucune raison de fermer! C'est le profit! ... détruire des vies et des familles. »

(Yoann Perrès, agent de production, 31 ans, neuf ans d'ancienneté, posté en 3/8.)

Les Conti ont ainsi remis au goût du jour un lexique de luttes des classes offensif, suggérant la spoliation, l'exploitation "des patrons», "des capitalistes», déplaçant la sémantique des injustices matérielles et morales subies - "les efforts, les sacrifices » des ouvriers non récompensés avant et après la fermeture de l'usine - sur le terrain des inégalités sociales - "l'enrichissement et le profit de quelques-uns » au détriment du plus grand nombre. Sans doute ces témoignages, deux ans après la fermeture, sont-ils portés par les salariés dont l'indignation perdure au fil du temps et que l'enquête, les sollicitant, participe aussi à reconstruire (Monjaret, 2005). Mais ils convoquent également une mémoire du conflit médiatisée par la figure de Xavier Mathieu. Ses discours se sont largement appuyés sur le registre d'une critique sociale fortement ancrée à gauche.

Il faut prendre ici la mesure du rôle tout à fait central de Roland Szpirko, qui ne s'est pas réduit à celui de conseil. Il a contribué à la production de cette parole radicale, qui s'est imposée au sein des Conti en lutte. Roland Szpirko a accompagné ce mouvement d'un travail militant, visant à qualifier l'expérience des rapports sociaux de classes et à l'inscrire dans l'histoire des luttes ouvrières. Les nombreuses démonstrations de force lors de manifestations(24) ont ainsi permis de faire exister ce conflit social en un conflit de classes au sens que

(24) De multiples manifestations se sont déroulées notamment à Paris, Compiègne, Beauvais, Sarreguemines, Hanovre, Amiens avec les salariés de Goodyear et plusieurs meetings ont été organisés sur le parking de l'usine, devant le palais de justice d'Amiens ou de Compiègne. lui donne Oscar Negt (2007). Il a incarné le retour de la révolte ouvrière dans un contexte, rappelonsle, favorable au mouvement social. La contestation exprimée dès janvier 2009 reste vive et prête à redescendre dans la rue. Ainsi, les manifestations du $1^{\text {er }}$ mai, cette année-là, de mémoire de syndicaliste, ont connu une affluence jamais atteinte dans la cité impériale :

«Il n'y a jamais eu de manifestation comme cela à Compiègne! C'était impressionnant. Moi, j'étais devant, j'avais la chair de poule. »

(Entretien avec Michel Bonte, délégué FO, réalisé en octobre 2013.)

"Quand j'ai vu le 1er mai, je me suis dit, c'est Mai 68 qui redémarre. Je pensais qu'on redémarrait. »

(Entretien avec Jacques Pichon, délégué CGT, réalisé en octobre 2013.)

L'expérience de l'action collective a été le support d'un processus de transformation sociale pour nombre d'individus qui y ont acquis une véritable conscience politique. Pour beaucoup, ce conflit a permis une découverte de la lutte collective, aiguisant une «subjectivité rebelle» (NEGT, 2007) qui a pu donner aux ouvriers le sentiment, durant le «temps chaud» du conflit, d'être à l'avant-garde d'un combat politique. Les Conti interviennent même en soutien d'autres salariés en lutte : "On est allés aider beaucoup de boîtes qui essayaient de s'accrocher au bateau. Tu arrivais avec cinq vestes Continental et le conflit était réglé tout de suite», se souvient Michel Bonte. Les usages que les Conti ont faits de leur veste de travail évoquent une résistance qui s'est forgée dans l'expérience de la lutte et figurent le renversement des rapports de domination. Ornée le plus souvent d'autocollants de la marque, cette veste n'a pas été le symbole du travail aliéné mais celui d'une identité rebelle qui cherche à recouvrer dans la lutte honneur et dignité de classe. Mais les Conti en ont été aussi prisonniers, comme le souligne Patrick Lachambre, délégué CGT en janvier 2014 :

"Cette image de Conti, on nous l'a foutue sur les épaules malgré nous si tu veux, car on nous a élevés un peu trop haut, je pense. On a fait notre boulot, on a fait le boulot qu'on devait faire. Je pense que c'est les autres qui n'ont pas fait leur boulot! Parce que sinon, ça n'arriverait pas ces licenciements. Mais il y en a beaucoup qui nous prennent pour beaucoup trop. Pour eux, ils ne nous prennent pas pour des dieux mais pas loin. »

Les Conti n'ont été que des porte-paroles d'une contestation sociale qui avait commencé début 2009 avec les journées nationales de mobilisation interprofessionnelle, mouvement resté trop éphémère, regrette Patrick Lachambre, pour peser réellement sur la gestion de la «crise» et offrir un véritable débouché politique. 


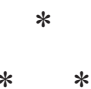

La lutte des Conti ne s'est pas arrêtée en juin 2009. Elle a connu de multiples rebondissements et se poursuit encore aujourd'hui dans une action engagée en premier lieu auprès du conseil des prud'hommes de Compiègne, qui réunit 678 plaignants. Le 31 août 2013, le groupe allemand Continental a été condamné pour défaut de motif économique et pour non-respect des obligations de reclassement. Le recours judiciaire des Conti, au-delà de la réparation financière, a un objectif d'ordre moral, celui de faire recouvrer aux ouvriers leur dignité par une décision de justice leur donnant raison. Leur lutte ainsi que ses modes opératoires et les discours militants expliquant sa légitimité ne s'appuient pas uniquement sur le caractère inhumain, immoral ou injuste de la perte d'emploi mais bien sur l'illégalité des procédures et des mesures de restructuration au regard du droit du travail, et sur les inégalités sociales qu'elles engendrent. Ce discours militant, peu observé et analysé, montre que l'identité collective et son expression politique n'ont pas totalement disparu sous l'effet des recompositions des classes populaires et du brouillage de plus en plus grand des messages politiques et syndicaux.

Cette page d'histoire sociale écrite par les Conti nous invite donc à porter une réflexion plus générale sur les transformations des classes sociales. Comme l'écrit Miguel Abensour (1988), elles «ne luttent pas parce qu'elles existent, elles en viennent à exister parce qu'elles luttent». Aujourd'hui encore, l'action collective est portée par le Comité de lutte qui, depuis la fermeture, continue de défendre les intérêts des anciens salariés. Les militants de la CGT et de FO ont dirigé pendant trois ans le comité d'entreprise qui a été dissous fin février 2014. Ils ont accompagné la grande majorité des plaignants dans la procédure judiciaire qui s'est poursuivie en avril 2014, avec l'audience en appel de la décision du conseil des prud'hommes de Compiègne. De son côté, la CFTC coordonne le recours judiciaire d'environ deux cents personnes qui continuent de lui faire confiance, comme le dit son représentant, José Munes. Les différends révélés durant le conflit restent vifs. Des tensions existent encore entre les courants syndicaux même si l'ancien responsable de la CFDT a quitté l'arène syndicale après la défaite aux élections professionnelles de 2010. Plus de la moitié des Conti est encore au chômage; certains sont en fin de droit. En l'absence de structures stables, l'enjeu a été de maintenir un collectif qui s'est révélé actif. Toutefois, les situations de chômage et de précarité, les mobilités professionnelles rendent de plus en plus aléatoires les rencontres entre Conti et creusent le lit de l'isolement. L'action prud'homale est un des derniers supports de la mobilisation qui, indubitablement, s'effrite.

\section{Bibliographie}

Abensour M. (1988), «La passion d'E. P. Thompson», préface de Thompson E. P., La formation de la classe ouvrière anglaise, Paris, Seuil, pp. I-XVI.

Askenazy P. (2011), Les décennies aveugles. Emploi et croissance 1970-2010, Paris, Seuil.

Beaud S., Pialoux M. (1999), Retour sur la condition ouvrière. Enquête aux usines Peugeot de SochauxMontbéliard, Paris, Fayard.

Béroud S., Mouriaux R. (2001), «Violence et sabotage dans les grèves en France», in Larose C., Cellatex, quand l'acide a coulé, Paris, Syllepse, pp. 141-167.

Béroud S., Denis J.-M., Giraud B., Pélisse J. (2008), «Une nouvelle donne ? Regain et transformation des conflits du travail», in Amossé T., Bloch-London C., Wolff L., Les relations sociales en entreprise, Paris, La Découverte, pp. 223-255.

Béroud S., Yon K (2009), «Face à la crise, que fait le mouvement social ? Quelques éléments de réflexion sur l'évitement relatif d'une stratégie de confrontation", Contretemps, $\mathrm{n}^{\circ} 3$ (nouvelle série), pp. 15-25.
Bourdieu P. (1984), Questions de sociologie, Paris, Éditions de Minuit.

Boltanski L., Chiapello È. (1999), Le nouvel esprit du capitalisme, Paris, Gallimard.

Burawoy M. (2011), «La domination est-elle si profonde ? Au-delà de Bourdieu et Gramsci», Actuel Marx, $\mathrm{n}^{\circ}$ 50, pp. 166-190.

Burgi-Golub N. (2002), «Exiler, désœuvrer les femmes licenciées », Travail, genre et sociétés, n 8, pp. 105-122.

Champagne P. (1984), «La manifestation. La production de l'événement politique», Actes de la recherche en sciences sociales, $\mathrm{n}^{\circ}$ 52-53, pp. 19-41.

Coutrot T. (1998), L'entreprise néolibérale, nouvelle utopie capitaliste?, Paris, La Découverte.

Depoorter P. (2006), Figures de la dignité ouvrière: enquête sur les usines Brissonneau-Chausson de Creil (1950-1996), Thèse de sociologie, université de Picardie.

Dif-Pradalier M., Reix F. (2012), Figures de salariés CFTC en lutte. Les cas de Continental et de Nortel, Pantin, CFTC. 
Didry C., Tessier L. (1996), «La cause de l'emploi», Travail et emploi, $\mathrm{n}^{\circ}$ 69, pp. 23-35.

FAJARDie F. (2003), Metaleurop, paroles ouvrières: entretiens avec des ouvriers de Metaleurop, Paris, Mille et une nuits.

FAssin D. (2009), «Les économies morales revisitées», Annales. Histoire, sciences sociales, ${ }^{\circ}$ 6, pp. 1237-1266.

FAvier I. (2008), Perrier-Nestlé, histoire d'une absorption: histoire sociale d'une entreprise à l'heure des changements culturels, 1990-2000, Ivry-sur-Seine, Éditions de l'Atelier.

FrASER N. (2005), Qu'est-ce que la justice sociale? Reconnaissance et redistribution, traduit de l'anglais par Estelle Ferrarese, Paris, La Découverte.

Goergen M.-L., Levesque C., Patillon C. (2007), Fermeture de boîtes... et après ?, Actes des rencontres de mars 2005 à la Maison des hommes et des techniques, Nantes, Éditions du Centre d'histoire du travail.

Honneth A. (2000), La lutte pour la reconnaissance, traduit de l'allemand par Pierre Rusch, Paris, Cerf.

Larose C., Béroud S. (collab.), Mouriaux R. (collab.), RabHi M. (collab.) (2001), Cellatex, quand l'acide a coulé, Paris, Syllepse.

LAZzARotTi R. (1968), L'industrie et les complexes industriels dans la vallée de l'Oise, étude de géographie économique et humaine, Gap, Imprimerie Louis Jean.

Linhart D., Rist B., Durand E. (2002), Perte d'emploi, perte de soi, Ramonville-Saint-Agne, Érès.

LinHART D. (2005), «D'un monde à l'autre : la fermeture d'une entreprise», Revue de l'Ires, n 47, pp. 81-94.

Magloire F. (2002), Ouvrière : récit, La Tour-d'Aigues, Éditions de l'Aube.

Malsan S. (2007), «Licenciements collectifs : le prix d'une dette symbolique», Revue du Mauss, $\mathrm{n}^{\circ} 29$, pp. 180-206.
Massera B., Grason D. (2004), Chausson : une dignité ouvrière, Paris, Syllepse.

Mathieu L. (2011), La démocratie protestataire. Mouvements sociaux et politique en France, Paris, Presses de Sciences Po.

Matonti F., Poupeau F. (2004), «Le capital militant. Essai de définition », Actes de la recherche en sciences sociales, $\mathrm{n}^{\circ} 155$, pp. 4-11.

Monjaret A. (2005), "Quand les lieux de travail ferment...», Ethnologie française, vol. 35, $\mathrm{n}^{\circ} 4$, pp. 581-592.

Montlibert (DE) C. (1989), Crise économique et conflits sociaux, Paris, L'Harmattan.

Negt O. (2007), L'espace public oppositionnel, traduit de l'allemand et préfacé par Alexander Neumann, Paris, Payot.

Pigenet M. (2012), «L'action directe et grève générale», in Pigenet M., Tartakowsky D., Histoire des mouvements sociaux en France, Paris, La Découverte, pp. 283-293.

Renault E. (2004), L'expérience de l'injustice, Paris, La Découverte.

Roupnel-Fuentes M. (2011), Les chômeurs de Moulinex, Paris, Presses universitaires de France.

Thompson E. P. (1988a), «L'économie morale de la foule», in Thompson E. P., Gauthier F., Ikni G. R., La guerre du blé au XVIII siècle : la critique populaire contre le libéralisme économique au XVIII siècle, Montreuil, Éditions de la Passion, pp. 31-92.

Thompson E. P. (1988b), La formation de la classe ouvrière anglaise, traduit de l'anglais par Gilles Dauvé, Mireille Golaszewski et Marie-Noëlle Thibault, Paris, Gallimard, Seuil.

Zimmermann B. (2011), Ce que travailler veut dire. Une sociologie des capacités et des parcours professionnels, Paris, Economica. 